\title{
The French press: A repeatable and high-throughput approach to exercising zebrafish (Danio rerio)
}

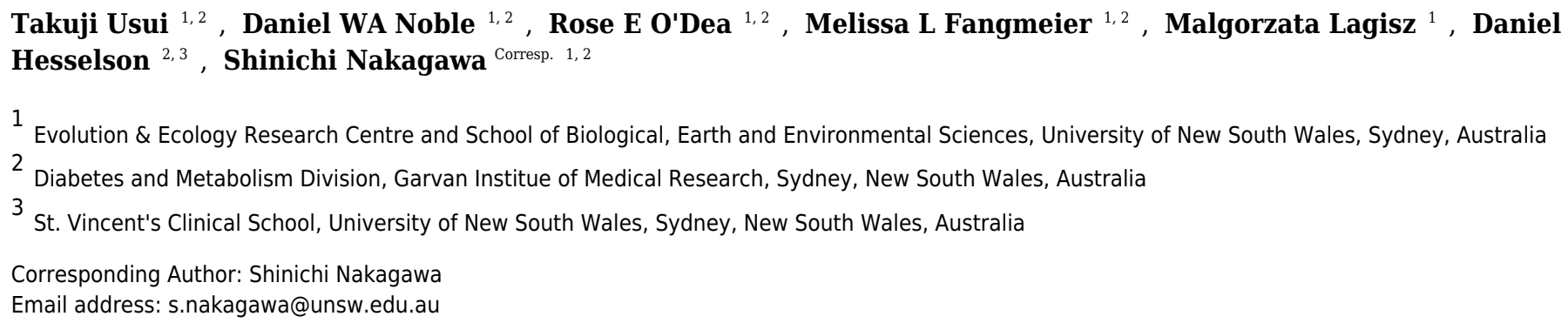

Zebrafish are increasingly used as a vertebrate model organism for various traits including swimming performance, obesity and metabolism, necessitating high-throughput protocols to generate standardized phenotypic information. Here, we propose a novel and costeffective method for exercising zebrafish, using a coffee plunger and magnetic stirrer. To demonstrate the use of this method, we conducted a pilot experiment to show that this simple system provides repeatable estimates of maximal swim performance (intra-class correlation $[I C C]=0.34-0.41$ ) and observe that exercise training of zebrafish on this system significantly increases their maximum swimming speed. We propose this highthroughput and reproducible system as an alternative to traditional linear chamber systems for exercising zebrafish and similarly sized fishes. 


\section{The French press: A repeatable and high-throughput approach}

\section{2 to exercising zebrafish}

3 Takuji Usui ${ }^{1,2}$, Daniel W.A. Noble ${ }^{1}$, Rose E. O'Dea ${ }^{1,2}$, Melissa L. Fangmeier ${ }^{1,2}$, Malgorzata Lagisz ${ }^{1}$, Daniel

4 Hesselson $^{2,3}$ and Shinichi Nakagawa ${ }^{1,2}$

$5{ }^{1}$ Evolution and Ecology Research Centre and School of Biological, Earth and Environmental Sciences,

6 University of New South Wales, Sydney, NSW 2052, Australia

$7{ }^{2}$ Diabetes and Metabolism Division, Garvan Institute of Medical Research, 384, Victoria Street,

8 Darlinghurst, Sydney, NSW 2010, Australia

$9{ }^{3}$ St. Vincent's Clinical School, University of New South Wales, Sydney, NSW 2052, Australia

10 Running title: Performance and exercise in fish

11 Corresponding author:

12 Shinichi Nakagawa ${ }^{1,2}$

13 Email address:

14 s.nakagawa@unsw.edu.au 


\section{Abstract}

Zebrafish are increasingly used as a vertebrate model organism for various traits including swimming performance, obesity and metabolism, necessitating high-throughput protocols to generate standardized phenotypic information. Here, we propose a novel and cost-effective method for exercising zebrafish, using a coffee plunger and magnetic stirrer. To demonstrate the use of this method, we conducted a pilot experiment to show that this simple system provides repeatable estimates of maximal swim performance (intra-class correlation $[\mathrm{ICC}]=0.34-0.41$ ) and observe that exercise training of zebrafish on this system significantly increases their maximum swimming speed. We propose this high-throughput and reproducible system as an alternative to traditional linear chamber systems for exercising zebrafish and similarly sized fishes.

\section{Introduction}

Zebrafish are used as a vertebrate model organism for diverse traits in biomedicine, developmental biology, behavioural science and evolutionary biology (Grunwald \& Eisen, 2002; Lieschke \& Currie, 2007; Norton \& Bally-Cuif, 2010). Central to many of these studies is the ability to easily and reliably exercise zebrafish and assay swimming performance (Palstra et al., 2010; Blazina, Vianna \& Lara, 2013; Gilbert, Zerulla \& Tierney, 2014), especially given that zebrafish models are being increasingly used for understanding the effects of exercise and obesity on human health (Seebacher et al., 2017). Furthermore, exercise systems are essential for studies on metabolic performance, where an organism's maximal metabolic rate (MMR) is measured after a period of exhaustive exercise (Killen, Norin \& Halsey, 2016). It is therefore desirable to 
36 have high-throughput and reproducible systems to assess zebrafish swim performance and

37 administer defined exercise regimens.

38 Common methods to exercise zebrafish include manually chasing fish until exhaustion (Ferguson

$39 \&$ Tufts, 1992) or traditional Brett-style swimming chambers in which individual fish swim

40 against increasing near-laminar water currents (Brett, 1964). These methods are, however, limited

41 by technical requirements of the protocol, low-throughput and/or high cost. For example, studies

42 using exercise respirometry in Brett-style swim chambers typically measure one or two fish per

43 day (Killen, Norin \& Halsey, 2016). More recent methods, in which zebrafish are forced to swim

44 until exhaustion in a beaker against increasing currents created by a rotating magnetic stir bar

45 (Blazina, Vianna \& Lara, 2013) generate a large central water vortex, limiting repeatability

46 between experiments.

47 Here, we present a high-throughput and cost-effective exercise system that uses a coffee plunger

48 to dramatically reduce vortex formation, and illustrate its application to maximal swim

49 performance measurements and exercise training. By reducing, without eliminating, vortex

50 formation we retain the ability to easily score exhaustion during maximal swim performance

51 trials. Our main aim is to demonstrate the use of this new high-thoughput system by conducting a

52 pilot study. In this pilot experiment, we assessed (i) intra-class correlation, ICC (in this case,

53 'intra-individual' correlation or repeatability; Nakagawa \& Schielzeth, 2010), in estimates of

54 maximum swimming speed, and (ii) whether maximum swimming speed increases after exercise

55 training (e.g. aerobic training paradigm). We additionally investigate whether (iii) maximum 
56 swimming speeds differed with sex and length of zebrafish, and (iv) if the mass of zebrafish

57 changed after exercise training.

\section{Materials \& Methods}

\section{Zebrafish and maintenance}

60 We used $40($ male $=20$; female $=20)$ wildtype zebrafish $($ Danio rerio $)$ that were approximately 6

61 months old (see below for the rationle for our sample size). Sex was determined from

62 morphology and confirmed with individual egg laying records. Zebrafish were housed in two 3.5

63 L plastic aquaria supplied with system water $(\mathrm{pH}=7.0$ to 8.0 ; water temperature $=27.0$ to 29.0

$64{ }^{\circ} \mathrm{C}$ ) via a filtration system, and placed in a room with a $14.5 \mathrm{~h}$ light: $9.5 \mathrm{~h}$ dark cycle. Fish were

65 fed once daily in the evening with commercial pellet food (O.range INVE Aquaculture). All

66 experiments were performed with the approval of the Garvan Institute of Medical Research

67 Animal Ethics Committee (Approval number 15/15).

\section{French press system setup}

69 We set up French press exercise units as illustrated in Figure 1. Zebrafish swam in the top compartment of the coffee plunger (IKEA Upphetta Coffee/tea maker; $400 \mathrm{~mL}$ volume; $8 \mathrm{~cm}$

71 diameter) against circular water currents created by a rotating magnetic stir bar ( 45 x 7 mm; Fig.

72 1A). We placed coffee plungers on top of single-plate (Labtek Magnetic Stirrer Hotplate; Fig. 1B)

73 and multi-plate (Labtek 10 Place Stirrer; Fig. 1C) magnetic stirrers for maximal swim

74 performance trials and exercise training, respectively. Since swim performance is shown to be

75 sensitive to water temperature (Wardle, 1980), we recorded water temperature for each trial and 
76 replaced water as necessary between trials to keep water temperature constant (average water

77 temperature across trials $=25.5 \pm 0.1^{\circ} \mathrm{C}$ ). Full protocols and a video illustration of the system are

78 available in the Supplementary Information.

79 Preliminary trials indicated that the water speed in the zebrafish swimming compartment was

80 substantially slower than the set speed of the magnetic stirrer. We thus determined actual water

81 speeds experienced by the zebrafish by tethering a freely rotating plastic leaf above the plunger

82 and determined its rpm for a range of stirrer speeds $(200-1400 \mathrm{rpm})$. Stirrer speed and water

83 speed exhibited a linear relationship (Fig. S3).

\section{Maximal swim performance}

85 We measured individual maximum swimming speeds of 40 adult zebrafish. We gradually

86 increased the stirrer speed at $10 \mathrm{rpm}$ steps every 5 seconds until maximum swimming speed,

87 defined as the speed at which zebrafish lost their ability to maintain their position in the water

88 column. Importantly, our step duration was long enough to observe the recovery of zebrafish that

89 were temporarily swept up into the water vortex at sub-maximal speeds, and thus avoid

90 underestimation of maximum swimming speeds. To assess individual repeatability and the effect

91 of exercise training, maximum swimming speed was measured twice for each zebrafish (i.e. pre-

92 and post-exercise training; an outline of the experimental design is shown in Fig. S4). One day

93 after the first round of maximal swim performance trials, each zebrafish $(n=40)$ was randomly

94 assigned to a control $(n=20)$ or experimental group $(n=20)$ that were matched for pre-

95 intervention maximum swimming speeds. Both our first and second round of maximal swim

96 performance trials were conducted blind to the non-exercised (control) and exercised 
97 (experimental) groups. All maximal swim performance trials were conducted between 9 am and 3

$98 \mathrm{pm}$.

99 Body mass and length of each zebrafish were also measured before each of the two maximal

100 swim performance trials. We weighed individual zebrafish by placing each fish into a small,

101 transparent holding container filled with system water and placed atop a measuring scale

102 (OHAUS Adventure Pro Precision Electronic Balance). To measure length, the holding container

103 was then placed on top of graphing paper $\left(1 \mathrm{~mm}^{2}\right.$ grid squares $)$ and photographs of each fish were

104 taken directly from above with a digital camera (Canon PowerShot SX710HS). The body length

105 of each zebrafish was estimated by calibrating individual photographs using ImageJ (version 1.51

106 k; Schneider, Rasband \& Eliceiri, 2012).

\section{Exercise training}

108 To investigate the effects of exercise training, the experimental group received a 40-minute

109 swimming regime at a sub-maximal exercise speed (stirrer speed $=\sim 370 \mathrm{rpm}$ ) for five

110 consecutive days using multi-plate magnetic stirrers. The control group was placed in identical

111 French press units for the same duration without any forced exercise (stirrer speed $=0 \mathrm{rpm}$ ).

112 Exercise training aimed to improve swimming ability (Brett, 1964; LeMoine et al., 2010) and the

113 sub-maximal swimming speed was determined during preliminary trials as the highest tolerated

114 continuous swimming speed that did not induce exhaustion within 40 minutes. All exercise

115 training trials were conducted at 9 am. 
117 Our sample size of 40 fish $($ male $=20$; female $=20)$ was determined by balancing logistical

118 considerations (e.g. the use of 10-plate mangenetic stirrer, meaning 10 fish can be exercised at

119 one time) and with reasonable power. Our design only allowed us to detect medium to large

120 effects (Cohen's $d=0.45$; note $d=0.3,0.5$ and 0.8 represents small, medium and large effects;

121 sensu Cohen, 1988) setting the power at 0.8 and with the alpha level of 0.05. All power

122 caculations were conducted using the pwr package (Champely, 2017).

123 We estimated repeatability (ICC estimates) in maximum swimming speeds using the rpt function

124 in the package $r p t R$, which estimates repeatability using a linear mixed-effects model (LMM)

125 framework (Stoffel, Nakagawa \& Schielzeth, 2017). Repeatability $(R)$ describes the proportion of

126 among-group ( $\sigma_{\alpha}^{2}$ ) variance out of the total variance (Sokal \& Rohlf, 1995):

$$
R=\sigma_{\alpha}^{2} /\left(\sigma_{\alpha}^{2}+\sigma_{\varepsilon}^{2}\right)
$$

We obtained three different estimates of repeatability, namely unadjusted, adjusted, and enhanced agreement repeatabilities, using separate models for each. Unadjusted repeatability (also referred to as agreement or broad sense repeatability; Shrout \& Fleiss, 1979) does not control for variance explained by fixed effects in the repeatability estimate, and in our case, only included individual fish ID as a random effect. Moreover, adjusted repeatability accounts for fixed effects by excluding the variance explained by fixed effects from the repeatability estimate, whilst enhanced agreement repeatability accounts for fixed effects by including their variance in the estimate 
136 length of zebrafish, as well as an interaction between treatment and measure as our fixed effects

137 (see Table S1 for coding of our variables). Uncertainty in repeatability estimates was obtained 138 using parametric bootstrapping, which was set to 10000 for all models.

139 To analyse the effect of exercise training on maximum swimming speed, we constructed linear 140 mixed-effects models using the lmer function in the lme4 package (Bates et al., 2015) fitting 141 individual fish IDs as a random effect. For fixed effects, we fitted an interaction between 142 treatment and measure to assess whether exercise training increased swim speed in the second 143 round of the maximum swim performance trial. We further included as fixed effects sex and 144 length of individual fish (to investigate differences in maximum swim speed with zebrafish sex 145 and length) and water temperature (to control for any differences due to water temperature).

146 Length and temperature variables were mean-centered prior to analysis. To assess change in mass 147 after exercise training, in a separate model we fitted sex of zebrafish (to account for sex 148 differences in mass), and an interaction between treatment and measure as fixed effects. We 149 assessed statistical significance (at the alpha level =0.05) using Scatterthwaite's approximation 150 for degrees of freedom obtained from the package lmerTest. Confidence intervals for variables 151 were obtained using the confint function provided in the lme4 package. All statistical analyses 152 were conducted in the R programming language (v 3.2.2; R Development Core team, 2016; see 153 Supplementary Information for data and analyses).

\section{Results}

Maximum swimming speed significantly increased in the exercise-trained group, but was unchanged in the control group (Exercise: $t=2.67, d f=37.87, P=0.011$; Control: $t=1.15, d f=$ 
158

159

160

161

162

163

164

165

166

167

168

169

170

171

172

173

174

175

176

177

consistency of phenotypes and measurement accuracy (Nakagawa \& Schielzeth, 2010; Stoffel, Nakagawa \& Schielzeth, 2017). We observed moderate ICC estimates of maximum swimming speeds after accounting for differences between control and treatment groups (point estimate range: $0.34-0.41$; Fig. 3).

We found weak evidence that maximum swimming speed was impacted by the sex or the length of zebrafish (Table 1). We also did not find differences in mass for the exercise-trained group compared to the control, although mass decreased slightly in the second measure of exhaustive exercise relative to the first $(t=-2.15 ; d f=38.00 ; P=0.038$; Table S2).

\section{Discussion}

Our main aim in this study was to demonsrate a new method that can be used for exersicing and measuring swim performance in zebrafish. Protocols to exercise and assess swim performance in zebrafish have broad applications including studies of human disease (Lieschke \& Currie, 2007), metabolism (Killen, Norin \& Halsey, 2016) and behaviour (Norton \& Bally-Cuif, 2010). In our pilot study, we demonstrated that we could obtain repeatable estimates of maximal swim performance. The use of a multi-plate magnetic stirrer array further enabled the high-throughput administration of an exercise regimen in a standardized manner. Additionally, we found exercise training to increase maximum swimming speed, which may reflect improved swimming capacity due to physiological and/or behavioural plasticity (Davison, 1997; Sinclair et al., 2014). We acknowledge that our pilot experiment had low power to detect any effects below $d=0.45$. Therefore, interest in sex and size effects warrant further investigation. Further, our finding of 
178 179

exercise eliciting an increase in swim performance should be replicated in the future when researchers employ our proposed method.

The simplicity of our maximal swim performance protocol allowed an observer to simultaneously test two zebrafish. With a 10-plate set-up, for example, the maximum swimming speed of $\sim 240$ zebrafish can be assessed in 5 hours with three observers ( $\sim .5$ minutes for each measurement). The duration of our maximal swim performance test falls between commonly defined $U_{\text {crit }}$ and burst swimming performance tests (Brett, 1964; Tierney, 2011), and thus swimming capacity as captured in our protocol may reflect a mixture of anaerobic and aerobic swimming abilities. By adjusting the magnitude and duration of flow speed it is possible to capture swimming abilities underlying different metabolic, physiological and ecological interests (Tierney, 2011). Our French press exercise system provides additional flexibility through adjustable temperature control settings. Understanding temperature related effects on exercise and swimming performance may be particularly useful in the context of organismal responses to recent climate change (Clark, Sandblom \& Jutfelt, 2013).

While our high-throughput and cost-effective protocol has clear advantages over current methods, it has three potential limitations. First, water speed varies across the radius of the coffee plunger potentially causing heavier fish to lose control at lower speeds than in a comparable linear swimming chamber (Rummer et al., 2016). This process would contribute a mass and position specific error to a given trial, possibly reducing repeatability. Despite this, we obtain moderate and significantly repeatable ICC estimates that are comparable to previous estimates of average repeatability in behavioural traits (Bell, Hankison \& Laskowski, 2009; Holtmann, Lagisz 
$199 \&$ Nakagawa, 2017), suggesting that this error is unlikely to compromise the utility of the system

200 as a general method to quantify maximal swimming performance. Moreover, similar repeatability

201 estimates obtained from both unadjusted and adjusted repeatability models suggest mass specific

202 error to be negligible. Matching the size of the French press swimming chamber to the particular

203 species of interest may also help reduce position specific error. Second, imbalanced use of one

204 side of the fish musculature due to circular swimming may result in premature, rather than

205 complete, exhaustion (Nilsson et al., 2007). Swim training in both clockwise and anti-clockwise

206 directions, as permitted with many standard magnetic stirrers, may be required to test swim

207 performance for both sides of the fish musculature. Finally, when considering endpoints related to

208 swim performance, it is important to consider that the kinematics of circular swimming will be

209 different than that of swimming in linear systems (Drucker \& Lauder, 1999). Nonetheless, the

210 simplicity of the proposed exercise system and its compatibility with multi-plate magnetic stirrers

211 allows for a high-throughput and repeatable exercise protocol in zebrafish, and offers an

212 affordable alternative to traditional linear systems.

\section{Conclusion}

214 In this study, we have presented a novel and high-throughput fish exercise system that provides

215 several advantages over using traditional linear chamber systems. Through the use of a coffee

216 plunger and magnetic stirrer, we are able to run a variety of exercise regimes with relative ease

217 and obtain repeatable and comparable estimates of swim performance. We propose that this

218 simple and reproducible method will be useful in a variety of research fields increasingly using

219 zebrafish and other species of fish for exercise, including in medical, metabolic and behavioural

220 research. 
221

222 223

224

225 226 227

\section{Acknowledgements}

We thank the staff at Garvan Institute of Medical Research for their support and general husbandry of zebrafish. We additionally thank Harry G Thomas for help during trials.

\section{Data Availability}

The raw data is available in the Supplementary Information.

\section{Supporting Information}

Supplementary information for this article can be found online.

\section{References}

Bates D, Mächler M, Bolker B, Walker S. 2015. Fitting linear mixed-effects models using lme4. Journal of Statistical Software 67:1-48. DOI 10.18637/jss.v067.i01.

Bell AM, Hankison SJ, Laskowski KL. 2009. The repeatability of behaviour. Animal Behaviour 77:771-783 DOI 10.1016/j.anbehav.2008.12.022.

Blazina AR, Vianna MR, Lara DR. 2013. The spinning task: a new protocol to easily assess motor coordination and resistance in zebrafish. Zebrafish 10:480-485 DOI $10.1089 /$ zeb.2012.0860.

Brett JR. 1964. The respiratory metabolism and swimming performance of young sockeye salmon. Journal of the Fisheries Research Board of Canada 21:1183-1226 DOI 10.1139/f64-103. 
238 Champely S. 2017. Package: pwr. Basic Functions for Power Analysis. R package version 1.2-1.

239 https://CRAN.R-project.org/package=pwr.

240 Clark TD, Sandblom E, Jutfelt F. 2013. Aerobic scope measurements of fishes in an era of 241 climate change: respirometry, relevance and recommendations. Journal of Experimental Biology 242 216:2771-2782 DOI 10.1242/jeb.084251.

243 Cohen J. 1988. Statistical Power Analysis for the Behavioral Sciences. Hillsdale, NJ: Lawrence 244 Erlbaum Associates.

245 Davison W. 1997. The effects of exercise training on teleost fish, a review of recent literature. 246 Comparative Biochemistry and Physiology 117A:67-75 DOI 10.1016/S0300-9629(96)00284-8.

247 Drucker EG, Lauder GV. 1999. Locomotor forces on a swimming fish: three-dimensional vortex 248 wake dynamics quantified using digital particle image velocimetry. Journal of Experimental 249 Biology 202:2393-2412.

250 Ferguson RA, Tufts BL. 1992. Physiological effects of brief air exposure in exhaustively 251 exercised rainbow trout (Oncorhynchus mykiss): implications for catch and release fisheries.

252 Canadian Journal of Fisheries and Aquatic Sciences 49:1157-1162 DOI 10.1139/f92-129.

253 Gilbert MJH, Zerulla TC, Tierney KB. 2014. Zebrafish (Danio rerio) as a model for the study of

254 aging and exercise: physical ability and trainability decrease with age. Experimental Gerontology 255 50:106-113. DOI 10.1016/j.exger.2013.11.013.

256 Grunwald DJ, Eisen JS. 2002. Headwaters of the zebrafish - emergence of a new model 257 vertebrate. Nature Reviews Genetics 3:717-724 DOI 10.1038/nrg892. 
258 Holtmann B, Lagisz M, Nakagawa S. 2016. Metabolic rates, and not hormone levels, are likely

259 mediator of between-individual differences in behaviour: a meta-analysis. Functional Ecology

260 31:685-696 DOI 10.1111/1365-2435.12779.

261 Killen SS, Norin T, Halsey LG. 2016. Do method and species lifestyle affect measures of

262 maximum metabolic rate in fishes? Journal of Fish Biology 90:1037-1046 DOI

$26310.1111 / \mathrm{jfb} .13195$.

264 LeMoine CMR, Craig PM, Dhekney K, Kim JJ, McClelland GB. 2010. Temporal and spatial

265 patterns of gene expression in skeletal muscles in response to swim training in adult zebrafish

266 (Danio rerio). Journal of Comparative Physiology B 180:151-160. DOI 10.1007/s00360-009-

$267 \quad 0398-5$.

268 Lieschke GJ, Currie PD. 2007. Animal models of human disease: zebrafish swim into view.

269 Nature Reviews Genetics 8:353-367 DOI 10.1038/nrg2091.

270 Nakagawa S, Schielzeth H. 2010. Repeatability for Gaussian and non-Gaussian data: a practical

271 guide for biologists. Biological Reviews 85:935-956 DOI 10.1111/j.1469-185X.2010.00141.x.

272 Nilsson GE, Nilsson SÖ, Penfold R, Grutter AS. 2007. From record performance to hypoxia

273 tolerance: respiratory transition in damselfish larvae settling on a coral reef. Proceedings of the

274 Royal Society B: Biological Sciences 274:79-85 DOI 10.1098/rspb.2006.3706.

275 Norton W, Bally-Cuif L. 2010. Adult zebrafish as a model organism for behavioural genetics.

276 BMC Neuroscience 11:90 DOI 10.1186/1471-2202-11-90.

277 Palstra AP, Tudorache C, Rovira M, Brittijn SA, Burgerhout E, van den Thillart GEEJM, Spaink

278 HP, Planas JP. 2010. Establishing zebrafish as a novel exercise model: swimming economy,

279 swimming-enhanced growth and muscle growth marker gene expression. PloS ONE 5:e14483

280 DOI 10.1371/journal.pone.0014483. 
281 R Development Core Team. 2016. R: a language and environment for statistical computing.

282 Vienna: R Foundation for Statistical Computing. Available at http://www.Rproject.org/

283 Rummer JL, Binning SA, Roche DG, Johansen JL. 2016. Methods matter: considering

284 locomotory mode and respirometry technique when estimating metabolic rates of fishes.

285 Conservation Physiology 4:1-13 DOI 10.1093/conphys/cow008.

286 Schneider CA, Rasband WS, Eliceiri KW. 2012. NIH Image to ImageJ: 25 years of image

287 analysis. Nature Methods 9, 671-675. DOI 10.1038/nmeth.2089.

288 Seebacher F, Tallis J, McShea K, James RS. 2017. Obesity-induced decreases in muscle

289 performance are not reversed by weight loss. International Journal of Obesity 41:1271-1278 DOI

$290 \quad \underline{10.1038 / \text { ijo.2017.81. }}$.

291 Shrout PE, Fleiss JL. 1979. Intraclass correlations: uses in assessing rater reliability.

292 Psychological Bulletin 86:420-428 DOI 10.1037/0033-2909.86.

293 Sinclair ELE, De Souza CRN, Ward AJW, Seebacher F. 2014. Exercise changes behaviour.

294 Functional Ecology 28:652-659 DOI 10.1111/1365-2435.12198.

295 Sokal RR, Rohlf FJ. 1995. Biometry: the principles and practice of statistics in biological

296 research. 3rd ed. New York: WH Freeman and Company.

297 Stoffel MA, Nakagawa S, Schielzeth H. 2017. rptR: repeatability estimation and variance

298 decomposition by generalized linear mixed-effects models. Methods in Ecology and Evolution

299 DOI 10.1111/2041-210X.12797.

300 Tierney KB. 2011. Swimming performance assessment in fishes. Journal of Visualized

301 Experiments 51:e2572. DOI 10.3791/2572. 
302 Wardle CS. 1980. Effects of temperature on the maximum swimming speed of fishes. In: Ali MA, 303 ed. Environmental physiology of fishes. New York: Springer Science+Business Media, 519-531.

304 Figure 1 French press exercise system setup for maximal swim performance trials and 305 exercise training of zebrafish. (A) Diagram of French press unit setup. Coffee plungers were $30675 \%$ filled with water and placed on a magnetic stirrer. Zebrafish swam against water currents 307 (yellow arrow) generated by a rotating magnetic stir bar (blue arrow). Plunger height was 308 standardized within and between trials by propping up the plunger using a vertical pipette tip 309 (grey triangle). (B) A single-plate coffee plunger set up for quantifying maximum swimming 310 speed. The stirrer speed was increased incrementally until maximum swimming speed was 311 reached (range: 360 - $910 \mathrm{rpm}$ ) (C) Multi-plate coffee plunger setup for multiplexed exercise 312 training. Up to ten zebrafish were simultaneously exercised in separate coffee plungers at a 313 submaximal speed.

(non-exercised) groups. Violin plots show the distribution of estimates on individual maximum swim speed estimates obtained from the first (red) and second (green) round of maximum swim performance trials for both experimental and control groups (sample sizes (n) are given for each group below violin plots). Arrows inside the violin plots represent mean estimate of maximum swim speeds and their $95 \%$ confidence intervals, as obtained from linear mixed-effects models.

Maximum swim speed increased significantly in the second round (green) of maximal swim performance trials compared to the initial round (red) in the exercise trained (experimental) group $(t=2.67, d f=37.87, P=0.011)$. Swim speed also increased slightly in the non-exercised control group, although this increase was not statistically significant $(t=1.15, d f=40.71, P=0.256)$. 
324 Figure 3 Forest plot of repeatability estimates in maximum swimming speed. Repeatability

325 estimates (circles) obtained from mixed-effects models are plotted with $95 \%$ confidence intervals

326 (lines). Estimates of unadjusted (0.34, 0.04 to 0.58$)$, adjusted $(0.41,0.16$ to 0.68$)$ and enhanced

327 agreement $(0.37,0.14$ to 0.60$)$ repeatabilities (or intra-class correlations, ICC) show that

328 maximum swimming speed is significantly repeatable. 


\section{Figure 1}

French press exercise system setup for maximal swim performance trials and exercise training of zebrafish.

(A) Diagram of French press unit setup. Coffee plungers were $75 \%$ filled with water and placed on a magnetic stirrer. Zebrafish swam against water currents (yellow arrow) generated by a rotating magnetic stir bar (blue arrow). Plunger height was standardized within and between trials by propping up the plunger using a vertical pipette tip (grey triangle). (B) A single-plate coffee plunger set up for quantifying maximum swimming speed. The stirrer speed was increased incrementally until maximum swimming speed was reached (range: 360 - 910 rpm) (C) Multi-plate coffee plunger setup for multiplexed exercise training. Up to ten zebrafish were simultaneously exercised in separate coffee plungers at a submaximal speed.

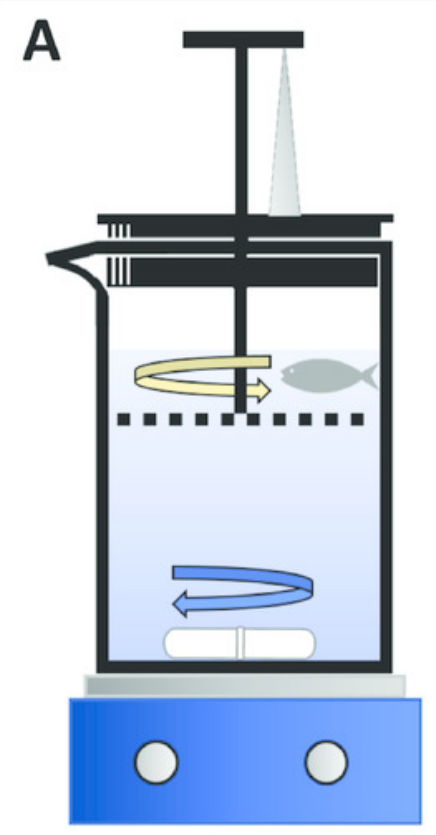

B

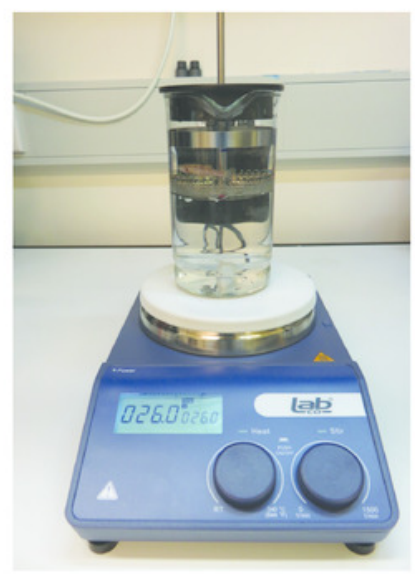

C

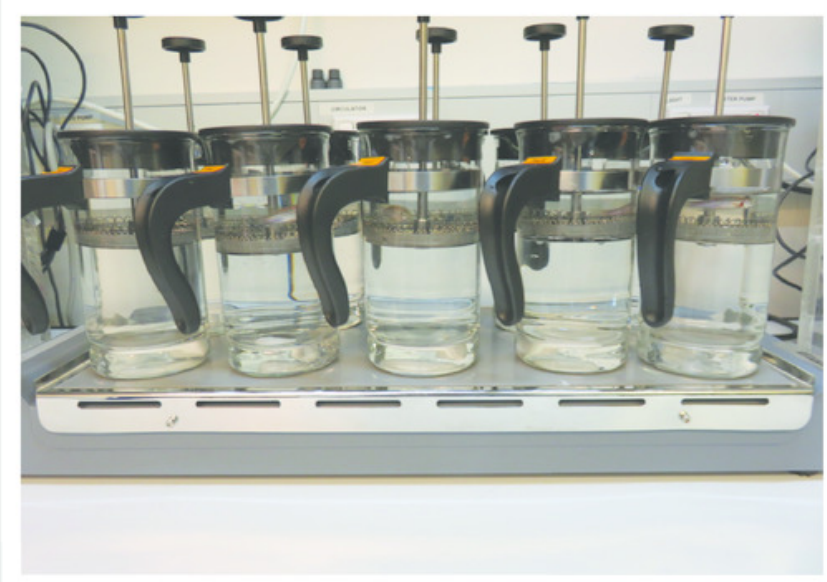




\section{Figure 2 (on next page)}

Violin plots of maximum swimming speed for experimental (exercised) and control (nonexercised) group

Violin plots show the distribution of estimates on individual maximum swim speed estimates obtained from the first (red) and second (green) round of maximum swim performance trials for both experimental and control groups (sample sizes $(n)$ are given for each group below violin plots). Arrows inside the violin plots represent mean estimate of maximum swim speeds and their $95 \%$ confidence intervals, as obtained from linear mixed-effects models. Maximum swim speed increased significantly in the second round (green) of maximal swim performance trials compared to the initial round (red) in the exercise trained (experimental) group $(t=2.67, d f=37.87, P=0.011)$. Swim speed also increased slightly in the nonexercised control group, although this increase was not statistically significant $(t=1.15, d f=$ $40.71, P=0.256)$. 


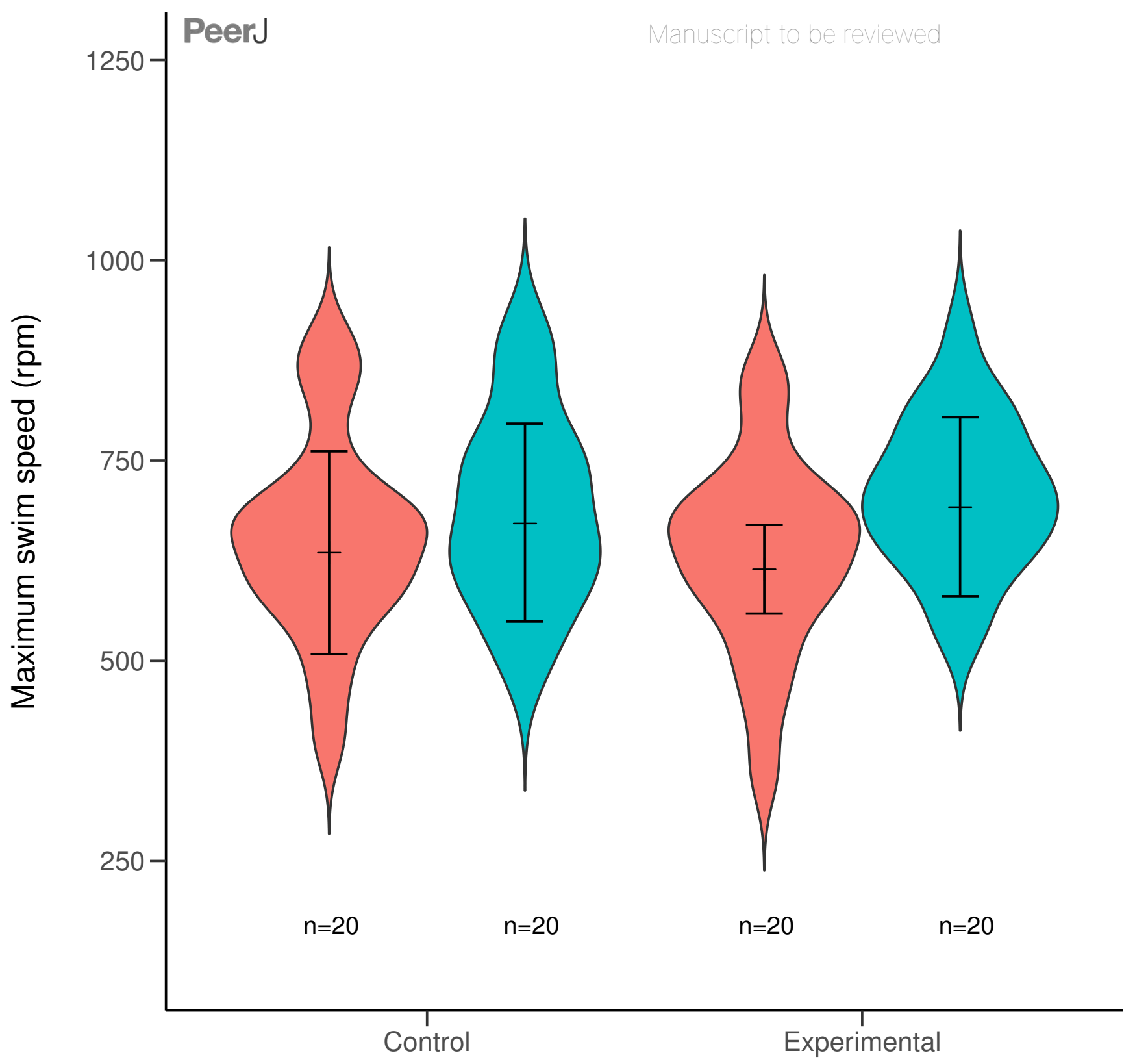


Figure 3 (on next page)

Forest plot of repeatability estimates in maximum swimming speed.

Repeatability estimates (circles) are plotted with $95 \%$ confidence intervals (lines). Estimates of unadjusted $(0.34,0.04$ to 0.58$)$, adjusted $(0.41,0.16$ to 0.68$)$ and enhanced agreement $(0.37,0.14$ to 0.60$)$ repeatabilities (or intra-class correlations, ICC) show that maximum swimming speed is significantly repeatable. 


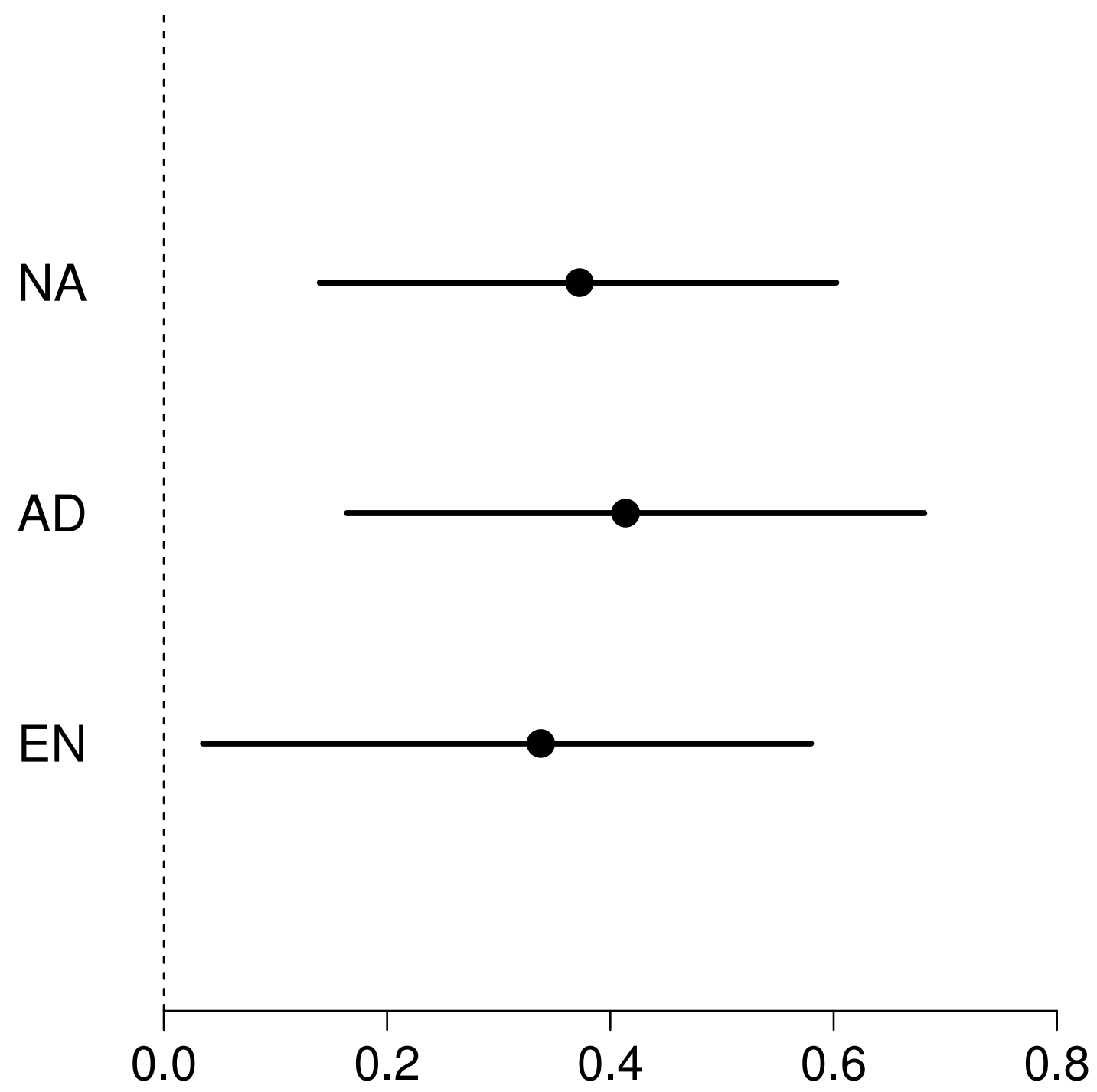




\section{Table $\mathbf{1}$ (on next page)}

Model coefficients from linear mixed-effects model investigating differences in maximum swimming speed (rpm).

Fixed effect intercept represents (i) first measure, (ii) experimental group, and (iii) female sex, mean-centered for zebrafish length and water temperature. Slope estimates, 95\% lower $(\mathrm{LCl})$ and upper (UCl) confidence intervals, $t$-values $(t)$, degrees of freedom ( $d f$ ) and P-values $(P)$ are reported. 
Table 1 Model coefficients from linear mixed-effects model investigating differences in

maximum swimming speed (rpm). Fixed effect intercept represents (i) first measure, (ii) experimental group, and (iii) female sex, mean-centered for zebrafish length and water temperature. Slope estimates, $95 \%$ lower (LCI) and upper (UCI) confidence intervals, $t$ values $(t)$, degrees of freedom $(d f)$ and P-values $(P)$ are reported.

\begin{tabular}{lcccccc}
\hline Fixed effects & Estimate & LCI & UCI & $t$ & $d f$ & $P$ \\
\hline Intercept & 614.3 & 558.9 & 669.7 & 20.982 & 55.90 & $<0.001$ \\
Measure (second) & 77.5 & 21.6 & 134.4 & 2.665 & 37.87 & 0.011 \\
Treatment (control) & 20.7 & -50.5 & 91.8 & 0.549 & 61.42 & 0.585 \\
Sex (male) & 34.3 & -26.5 & 95.2 & 1.070 & 35.37 & 0.292 \\
Length & 8.6 & -3.7 & 20.9 & 1.326 & 35.38 & 0.193 \\
Measure by treatment & -41.0 & -119.1 & 38.3 & -1.009 & 37.41 & 0.319 \\
(second*control) & -2.9 & -44.4 & 36.4 & -0.140 & 58.58 & 0.889 \\
Water temperature & Variance $\left(\sigma^{2}\right)$ & SD $(\sigma)$ & LCI $(\sigma)$ & UCI $(\sigma)$ \\
\hline Random effects & 5416 & 73.6 & 32.2 & 100.0 \\
\hline Individual fish ID & 7969 & 89.3 & 70.1 & 109.4 \\
\hline Residual & & & & & & \\
\hline
\end{tabular}

